Research Article

\title{
The Dynamic Broker Dominant Supply-Demand Matching Decision Using Multiagent System
}

\author{
Xu Zhang $\left(\mathbb{D}\right.$, Xu-mei Yuan $\left(\mathbb{D}\right.$, and Fu-li Wei ${ }^{1}$ \\ School of Economics and Management, Yanshan University, Qinhuangdao, Hebei, China \\ Correspondence should be addressed to Xu-mei Yuan; yxmysu@163.com
}

Received 2 December 2019; Revised 25 May 2020; Accepted 4 June 2020; Published 29 June 2020

Academic Editor: Francesco Clementi

Copyright (c) $2020 \mathrm{Xu}$ Zhang et al. This is an open access article distributed under the Creative Commons Attribution License, which permits unrestricted use, distribution, and reproduction in any medium, provided the original work is properly cited.

Supply-demand matching is critical for the increase of response speed, the improvement of resource utilization, and the building of long-term partnership. This study will contribute to the present supply-demand matching studies by (1) summarizing the dynamic broker dominant supply-demand matching problem (B-SDMP), (2) proposing the dynamic broker dominant supplydemand matching approach, inclusive of (a) the building of the distributed constraint satisfaction model based on the dynamic negotiation among agents and (b) the design of an asynchronous backtracking algorithm according to interaction among agents, and (3) the design and development of a multiagent dynamic supply-demand matching system. To verify the validity and usability of the method, the system test and case simulation are conducted. The matching solutions yielded from this B-SDMP analysis can help the buyers find the appropriate sellers on one commodity/service under dynamic environment and stimulate the building of long-term partnership among the sellers, buyers, and broker as a stable supply chain.

\section{Introduction}

Transactions refer to two-sided marketplaces in which both sellers and buyers raise their requirements for trading. The surge of market economy dramatically increases the amount of sellers, buyers, and information, and it is hard for the seller or buyer to find the matched partners and complete the transaction quickly. This has brought the opportunity to the broker. Besides, with the increased environmental uncertainty and complexity, the supply-demand matching decision based on the broker, i.e., dynamic broker dominant supply-demand matching, is becoming progressively reasonable.

Both practitioners and academia have stressed the supply-demand matching problem based on the broker, especially under the uncertain environment. Given the definition presented by Bhattacherjee [1], the dynamic broker dominant supply-demand matching problem is defined as "the broker who provides the optimal matching solutions considering the dynamic changes of buyers and sellers as well as their information." The existing relevant research studies can fall into two parts, i.e., broker dominant supply-demand matching and dynamic supply-demand matching.

The former part has been leaping forward in multiple areas, such as the existing matching decisions based on real estate agents, participated by own knowledge agents, according to financial institutions, decided by housekeepers and employers, etc. [2-5]. Jung and Jo [6] presented a twolayer multiagent framework between buyers and sellers for the broker and solved it using the constraint satisfaction problem model. Klerkx and Leeuwis [7] gave an overview of the mechanism of supply-demand matching decision from the perspective of innovation intermediaries. Jiang et al. [8] built a multiobjective matching optimization model taking the maximization of matching degree and trade volume as objectives and solved it using simulated annealing genetic algorithm. Liang and Jiang [9] considered the broker's attitude when researching two-sided matching problem. Amrutha et al. [10] and Zhang et al. [11] studied the supplydemand matching problem using the linear programming model for a low-carbon electricity system and a seaborne coal transaction project, respectively. Wang et al. [12] proposed a stable-matching-based method with user 
preference order for the problem of low accuracy of user linking in cross-media sparse data. Zhou et al. [13] proposed an agent-based resale model considering multibroker on China's resale housing market.

For the latter, the research of dynamic matching has a relatively late start and the most relevant studies are about supply-demand uncertainty. Zaefarian et al. [14] considered sellers should be able to match with buyers in dynamic environment. Wang et al. [15] classified and concluded the uncertainty in supply and demand and also studied the corresponding matching problem in uncertainty conditions. $\mathrm{Xu}$ [16] studied the evolution of the relationship between the supplier and the key customer from the dynamic matching perspective. Jiang et al. [17] studied dynamic supply-demand matching problem using the ideas and methods of multiagent system developed with computer language. Xu et al. [18] designed a dynamic scheduling model integrated with IoT which can optimize matching relation between transfer tasks and AGVs. Dastyar et al. [19] researched the relationship of suppliers and demanders under market dynamics.

Despite the headway made in these matching problems, there are fewer studies considering both of the noted two aspects. In the present paper, a model and its algorithm have been designed to solve the dynamic broker dominant supply-demand matching problem (B-SDMP). Furthermore, this paper developed an information system using multiagent system and Internet technology to achieve the dynamic matching.

The remainder of this paper is organized as follows. The definition and processes of dynamic broker dominant supply-demand matching problem are proposed in Section 2. The dynamic B-SDMP multiagent model is built in Section 3. An asynchronous backtracking algorithm is proposed in Section 4 to solve the model based on multiagent interaction. A multiagent system is developed in Section 5 for dynamic broker dominant supply-demand matching approach, including the model and its algorithm. Section 6 performs the system test and example simulation of the proposed dynamic matching system. Finally, some conclusions and future research problems are summarized in Section 7.

\section{Dynamic B-SDMP Description}

The critical parameters of the dynamic broker dominant supply-demand matching problem (B-SDMP) in this paper include the sellers' changing supply, buyers' random demand, and dynamic matching dominated by the broker. The dynamic broker dominant supply-demand matching decision processes are as follows:

(1) The buyers make an offer and provide their reservation price, ideal price, and multiattribute requirements (as supply quality, management quality, production capacity, operation scale, geographic position, traffic convenience, service level, corporate reputation, etc.) to the broker when needing one commodity
(2) The broker releases the buyers' demand information and reservation price to the sellers

(3) If the sellers have an intention to participate, they give their reservation prices, ideal price, and multiattribute value for the broker

(4) The broker maintains the above noted information, selects the qualified sellers based on the buyers' requirements, and provides the appropriate matching solutions that can ensure the stability of their cooperation

In these noted processes, reservation price is the upset price of sellers and the best bid of buyers. Besides, the ideal price is the ceiling price of sellers and the lowest price of buyers.

Given the features of dynamic B-SDMP and the actual requirement of matching solutions, the major research questions consist of distributed constraint satisfaction problem (DCSP), dynamic negotiation problem, and multiagent interaction problem, as shown in Figure 1.

(1) Distributed constraint satisfaction problem (DCSP): A DCSP is a distributed form of CSP. This distributed environment consists of multiple autonomous agents, with each one holding one or more variable. The DCSP is formally defined as follows:

$$
\begin{aligned}
& \text { A set of } n \text { agents: } A=\left\{A_{1}, A_{2}, \ldots, A_{n}\right\} . \\
& \text { A set of } m \text { variables of each agent } \\
& A_{i}: V=\left\{V_{1}, V_{2}, \ldots, V_{m}\right\} \text {, where each } V_{j} \text { pertains } \\
& \text { to one } A_{i}, \text { i.e., }\left(V_{j}, A_{i}\right) \text {. } \\
& \text { A set of domains of each variable: } \\
& D=\left\{D_{1}, D_{2}, \ldots, D_{m}\right\} \text {. } \\
& \text { A set of constrains: } C=\left\{C_{1}, C_{2}, \ldots, C_{n}\right\} \text {, where } \\
& \text { each } C_{i} \text { comprises a set of variables } \\
& V=\left\{V_{1}, V_{2}, \ldots, V_{m}\right\} \text { and a constrain } \\
& R \subseteq D_{1} \times D_{2} \times \ldots \times D_{m} \text {. If the value assignment of } \\
& \text { these variables satisfies this constrain, it returns to } \\
& \text { true. Otherwise, it returns to false. }
\end{aligned}
$$

In this study, the dynamic B-SDMP for selecting suppliers based on the buyers' multiattribute requirements can be considered as DCSP which takes the buyers' requirements as the domains and uses sellers' relevant data as the variables. By solving this DCSP, the broker can help each buyer find the sellers that satisfy all constraint conditions. In such a way, information sharing can be optimized, information transaction cost can be saved, and information security can be improved.

2.1. Dynamic Negotiation Problem. Dynamic negotiation refers to the activity of the agents to reach a consensus on their common concern issues. The dynamic negotiation problem can be defined as a ternary array $\{A, E, O\}$, where $A=\left\{A_{1}, A_{2}, \ldots, A_{n}\right\}$ denotes the set of agents participating in negotiation, $E$ is the negotiation strategies among the agents, and $O$ represents the negotiation behavior of each agent. The agents determine their own negotiation behavior in accordance with specific strategies and algorithms. The negotiation results are a group of independent collaboration 


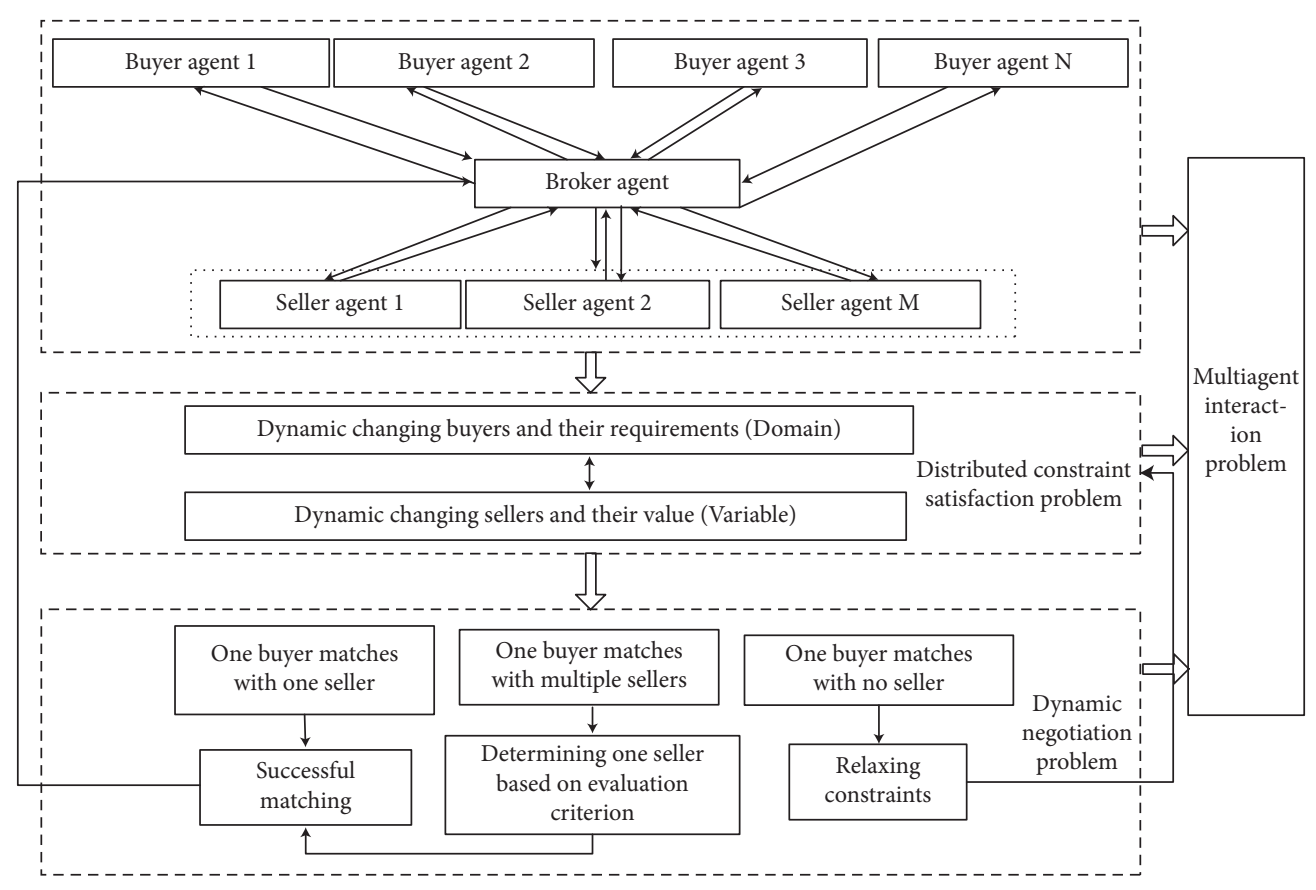

Figure 1: Ideas and problems of dynamic broker dominant supply-demand matching.

solutions among agents, which can help to eliminate conflicts and make joint decisions.

The matching solutions should be the one-to-many relationships according to the features of dynamic B-SDMP, i.e., one buyer cooperates with one seller for one commodity, and one seller is capable to providing products or services for more than one buyer. Yet, there can be several different situations, which include one solution, multiple solutions, or no solution for the above noted problem of distributed constraint satisfaction. For one solution, the matching solution can be determined and the matching processes can be completed. For multiple solutions or no solution, the matching processes continue until the seller for each buyer is appropriately matched by dynamic negotiation among the agents. These processes can be regarded as the dynamic negotiation problem in the B-SDMP.

2.2. Multiagent Interaction Problem. Information exchanging and interacting among the agents are indispensable for both the distributed constraint satisfaction problem and the dynamic negotiation problem. Communication, the necessary method to achieve the multiagent interaction, refers to the behavior of exchanging message among the agents. In this dynamic B-SDMP, the multiagent interaction behavior can be described as follows:

(1) The interaction behavior generated by data transmission, including demand type, time, quantity, reservation price, ideal price, and multiattribute value, among the broker agent and the buyer/seller agents

(2) The interaction behavior generated by the transaction of matching results among the broker agent and the buyer/seller agents when the matching succeeds
(3) The interaction behavior generated by dynamic negotiation among the broker agent and the buyer/ seller agents when the conflicts of matching occur

\section{Dynamic B-SDMP Multiagent Model}

The relationship between the buyers and sellers in two-sided matching is constrained. The aim of dynamic supply-demand matching is to seek out the appropriate seller whose multiattribute values can meet the buyer's constraint conditions. Besides, the dynamic B-SDMP can be considered as distributed constraint satisfaction problem under dynamic negotiation and abstracted to the corresponding model using multiagent system, as mentioned above.

In accordance with the theory of distributed constraint satisfaction and dynamic negotiation, the dynamic broker dominant supply-demand matching model in this paper is denoted as a hexahydric array $P=\langle A, G, V, D, S, O\rangle$, where

$A=\left\{A_{1}, A_{2}, \ldots, A_{n}\right\}$ denotes the set of seller agents

$G=\left\{G_{1}, G_{2}, \ldots, G_{m}\right\}$ is the set of buyer agents

$V=\left\{V_{1}, V_{2}, \ldots, V_{l}\right\}$ is the attribute value set of each seller agent $A_{i}, V \in A$, i.e., pertains to $(V, A)$

$D=\left\{D_{1}, D_{2}, \ldots, D_{l}\right\}$ the domain of $V$, is the attribute constrain set of each agent $G_{h}, D \in G$, i.e., pertains to $(D, G)$

$S($ belongs $(V, A))=\left\{\left(V_{1} \in D_{1}, V_{2} \in D_{2}, \ldots, V_{14} \in D_{14}\right)\right.$, belongs $\left.\left(D, G_{h}\right)\right\}$ is the dynamic matching solutions which includes all the seller agents $A$ which do not violate any constraints of buyer agent $G_{h}$

Yet, the number of elements in set $S$ can be zero, one, or more. This suggests that there may be zero, one, or multiple 
seller agents for one buyer agent $G_{h}$. It is required to determine the negotiation behavior of each seller agent $A_{i}$ and buyer agent $G_{h}$ under different situations.

\subsection{Negotiation Behavior}

3.1.1. Seller Agent Negotiation Behavior. Much of the real deal is buyer-conducted, and the negotiation behavior of sellers that is based only on the transaction price is comparatively simple. The negotiation behavior of seller agent $A_{i}$ can be written in equation (1), where $\mathrm{IP}_{A_{i}}$ and $\mathrm{RP}_{A_{i}}$ are the ideal and reservation price of seller agent $A_{i}$, respectively, $\mathrm{IP}_{G_{h}}$ and $\mathrm{RP}_{G_{h}}$ are similarly the ideal and reservation price of buyer agent $G_{h}$, and $P$ is the transaction price of each of the two:

$$
O\left(A_{i}\right)= \begin{cases}\text { Accept } G_{h}, & P \in\left[R P_{A_{i}}, I P_{A_{i}}\right] \cap\left[I P_{G_{h}}, R P_{G_{h}}\right] \\ \text { Quit, } & \text { otherwise. }\end{cases}
$$

(1) Satisfaction of buyers and sellers about price:

According to the reality, the sellers and buyers are sensitive to the transaction price, that is, the transaction price should not be lower than the reserved price, but should be as close as possible to the ideal price, Meanwhile, the sensitivity of different transaction prices varies from company to company. This paper assumes that the satisfaction of the sellers and buyers with respect to the transaction price is concave and convex, and the satisfactory function of the seller agent and buyer agent can be written as equations (2) and (3), respectively:

$f(P)= \begin{cases}0, & P<\mathrm{RP}_{A_{i}}, \\ \eta_{1}+\left(1-\eta_{i}\right)\left(\frac{P-\mathrm{RP}_{A_{i}}}{\mathrm{IP}_{A_{i}}-\mathrm{RP}_{A_{i}}}\right), & \mathrm{RP}_{A_{i}} \leq P \leq \mathrm{IP}_{A_{i}}, \\ 1 & P>\mathrm{IP}_{A_{i}} .\end{cases}$

$\delta(P)= \begin{cases}1, & P<\mathrm{IP}_{G_{h}}, \\ \theta_{h}+\left(1-\theta_{h}\right)\left(\frac{\mathrm{RP}_{G_{h}}-P}{\mathrm{RP}_{G_{h}}-\mathrm{IP}_{G_{h}}}\right), & \mathrm{IP}_{G_{h}} \leq P \leq \mathrm{RP}_{G_{h}}, \\ 0, & P>\mathrm{RP}_{G_{h}},\end{cases}$

where $\eta_{i}\left(0<\eta_{i}<1\right)$ and $\theta_{h}\left(0<\theta_{h}<1\right)$ denote the satisfaction of $A_{i}$ and $G_{h}$ when transaction price $P$ equals to their reservation price $\mathrm{RP}_{A_{i}}$ and $\mathrm{RP}_{G_{h}}$, respectively. Besides, $\beta_{i}\left(\beta_{i}>0\right)$ and $\alpha_{h}\left(\alpha_{h}>0\right)$ are the sensitive coefficient of $A_{i}$ and $G_{h}$ about the transaction price.

(2) Determination of transaction price:

Both buyers and sellers are susceptible to price, and the transaction is the result of the game equilibrium of these two sides. Thus, the transaction can be concluded if there is a satisfactory solution to both of them, i.e., $f(P)=\delta(P)$. Given the nature of equilibrium and the theorem of zero point existence, the properties of transaction price $P$ are as follows.

Properties 1. The seller agent $A_{i}$ and buyer agent $G_{h}$ can conclude the transaction at price $\mathrm{P}$ when $f\left(\mathrm{RP}_{A_{i}}\right) \leq \delta\left(\mathrm{RP}_{A_{i}}\right)$ and $f\left(\mathrm{RP}_{G_{h}}\right) \geq \delta\left(\mathrm{RP}_{G_{h}}\right)$

Properties 2. If the transaction price $P$ exists, it will be unique.

Proof. Let $F(P)=f(P)-\delta(P) \quad$ and $\quad F(P)=f\left(\mathrm{RP}_{A_{i}}\right)-$ $\delta\left(\mathrm{RP}_{A_{i}}\right) \leq 0$ when $P=\mathrm{RP}_{A_{i}}$ and $F(P)=f\left(\mathrm{RP}_{G_{h}}\right)-\delta\left(\mathrm{RP}_{G_{h}}\right) \geq 0$ when $P=\mathrm{RP}_{G_{h}}$. In accordance with the zero point existence theorem, there is a $P$ between $\mathrm{RP}_{A_{i}}$ and $\mathrm{RP}_{G_{h}}$, which makes $F(P)=f(P)-\delta(P)=0$, i.e., $f(P)=\delta(P)$. Then, $P$ denotes the transaction price of seller $A_{i}$ and buyer $G_{h}$.

There are three situations about $f(P)=\delta(P)$ in line with equations (2) and (3):

(i) $f(P)=\delta(P)=0$ : in this situation, the satisfaction of both $A_{i}$ and $G_{h}$ are zero and the transaction cannot be achieved.

(ii) $f(P)=\delta(P)=1$ : this situation suggests that $P \leq \mathrm{IP}_{G_{h}}$ and $P \geq \mathrm{IP}_{A_{i}}$. On the one hand, the buyers provide their ideal and reservation price earlier following the dynamic supply-demand matching process. On the other hand, the satisfactory function of the seller increases monotonously. Accordingly, the final transaction price $P$ refers to the buyer's ideal price $\mathrm{IP}_{G_{h}}$.

(iii) $0<f(P)=\delta(P)<1$ : this situation suggests that $\mathrm{IP}_{G_{h}}<P<\mathrm{RP}_{G_{h}} \quad$ and $\quad \mathrm{RP}_{A_{i}}<P<\mathrm{IP}_{A_{i}}$, i.e., $P \in\left[\mathrm{RP}_{A_{i}}, \mathrm{IP}_{A_{i}}\right] \cap\left[\mathrm{IP}_{G_{h}}, \mathrm{RP}_{G_{h}}\right]$. Thus, the transaction can be concluded.

Assume that there are two transaction price $P$ and $P^{\prime}$ on $\left[\mathrm{RP}_{A_{i}}, \mathrm{IP}_{A_{i}}\right] \cap\left[\mathrm{IP}_{G_{h}}, \mathrm{RP}_{G_{h}}\right]$, and $f(P)<f\left(P^{\prime}\right), \delta(P)>\delta\left(P^{\prime}\right)$, i.e., $f(P)<\delta\left(P^{\prime}\right)<\delta(P)$ for $f(P)$ and $\delta(P)$ are, respectively, monotonic increasing and decreasing about $P$. These results go against $f(P)=\delta(P)$. Thus, there is only one transaction price $P$ on $\left[\mathrm{RP}_{A_{i}}, \mathrm{IP}_{A_{i}}\right] \cap\left[\mathrm{IP}_{G_{h}}, \mathrm{RP}_{G_{h}}\right]$, which makes $f(P)=\delta(P)$.

Q.E.D.

In brief, if the transaction price is in the range of their ideal and reservation price, the seller $A_{i}$ will cooperate with buyer $G_{h}$ and return a message as "agreeing to match" to the broker. Otherwise, the seller $A_{i}$ will back out of the deal and match again with the inclined buyers in the waiting queue.

3.1.2. Buyer Agent Negotiation Behavior. The negotiation behavior of buyer agent $G_{h}$ can be expressed as follows in equation (4), where $|S|$ is the number of elements in set $S$ : 


$$
O\left(G_{h}\right)= \begin{cases}\text { Accept } A_{i}, & |S|=1, \\ \text { Accept } A_{i} \text { where } \max E\left(A_{i}\right), & |S|>1, \\ \text { relax } D, & |S|=0, \\ \text { Quit, } & \text { Otherwise }\end{cases}
$$

When searching for one sustainable seller only, i.e., $|S|=1$, the buyer agent $G_{h}$ could accept seller $A_{i}$ who meets its requirements and returns a message as "agreeing to match" to the broker. When searching for multiple sustainable sellers, i.e., $|S|>1$, the broker negotiates with the buyer based on the evaluation function $E\left(A_{i}\right)$ as equation (5), which will be affected by their own satisfaction with transaction price, preference order, and importance of seller, and the buyer $G_{h}$ can match with seller $A_{i}$ with the maximize evaluating value. Furthermore, when there is no seller, i.e., $|S|=0$, the broker negotiates with the buyer $G_{h}$ about whether or not he can relax his constraint $D$. Besides, the buyer $G_{h}$ exits the deal, backs again to the waiting queue, and waits the inclined sellers to match:

$$
\begin{aligned}
E\left(A_{i}\right) & =W_{1} * C_{G_{h} A_{i}}+W_{2} * T_{i} \\
& =W_{1} *\left(\mu_{1} * C P_{G_{h} A_{i}}+\mu_{2} * C R_{G_{h} A_{i}}\right)+W_{2} * T_{i},
\end{aligned}
$$

where $T_{i}$ denotes the importance of seller agent $A_{i}, C_{G_{h} A_{i}}$ is the satisfaction of buyer agent $G_{h}$ on seller $A_{i}$, and $C P_{G_{h} A_{i}}$ and $\mathrm{CR}_{G_{h} A_{i}}$ are the buyer's satisfaction of transaction price and preference ordinal, respectively. The $\mathrm{CP}_{G_{h} A_{i}}$ can be determined by equations (2) and(3) and the calculation method of transaction price in 3.3.1. There is no linear decreasing relationship between the preference order and satisfaction of buyers. The buyers are more concerned about their most preferred sellers, but less sensitive to the sellers ranking lower. Based on the method of [20], the satisfaction function about preference order of buyers, $\mathrm{CR}_{G_{h} A_{i}}$, is as follows:

$$
\mathrm{CR}_{G_{h} A_{i}}= \begin{cases}\left(\frac{I+1-\gamma_{h i}}{I}\right), & \gamma_{h i} \leq \varphi_{h}, \\ 0, & \text { otherwise, }\end{cases}
$$

where $I$ is the number of suppliers, $\gamma_{h i}$ is the preference order of suppliers $i$ for demand agent $h$, and $\varphi_{h}$ is the highest acceptable preference order of demand agent $h$.

\section{Dynamic B-SDMP Solution Algorithm}

The dynamic supply-demand matching problem can be seen as distributed constraint satisfaction problem (DCSP) as mentioned above, and the classic solution algorithms consist of asynchronous backtracking (ABT), asynchronous Weakcommitment search (ABS), distributed break out (DB), etc.

Asynchronous backtracking (ABT) serves as a pioneer algorithm to solve DCSP dating its first version from 1992 by Yokoo. ABT refers to an asynchronous algorithm performed autonomously by each agent in the distributed constraint network. Each agent takes its own decisions and informs other agents of them, and no agent has to wait for decisions of others. In ABT, the order of agent priorities is predetermined and identified by the alphabetical order. Each agent sends or receives the OK? and Nogood message and performs the check_agent_view operation. Therein, the OK? and Nogood messages are the assignment and conflicting assignments, respectively, and agent_view is the data structure holding by each agent which contains the most recent assignments received from agents with higher priority. The sample form of asynchronous backtracking algorithm is as follows. Each agent initializes its variables and informs its lower priority neighbors by sending them OK? message. If the agent cannot find an assignment that is consistent with the assignment of higher priority neighboring agents, it will send the inconsistent tuple to their agent_view with a Nogood message and eliminate this conflicting assignment from its current view.

In B-SDMP, the characteristics that the priorities order of buyers and sellers can be predetermined, and each buyer or seller agent knows its own variable value or constraint condition. These characteristics can meet the requirements of asynchronous backtracking algorithm. To solve the dynamic supply-demand matching problem, this paper proposes the asynchronous backtracking algorithm using the multiagent interaction method. The processes of this algorithm are as follows.

Step 1 : the buyer agents, respectively, set their constraint conditions $D$, ideal price $\mathrm{IP}_{G_{h}}$, reservation price $\mathrm{RP}_{G_{h}}$, demand time, quantity, etc.

Step 2 : the broker agent releases the approved buyer information including reservation price $\mathrm{RP}_{G_{h}}$, demand time, and quantity to the seller agents.

Step 3 : the seller agents submit their cooperation applications and provide the information of their multiattribute values for the intentional transaction.

Step 4 : the broker agent conducts the asynchronous backtracking search for the filtrating issues based on DCSP in dynamic B-SDMP at a regular time:

Step 4.1 : the importance of sellers and buyers are determined by the broker as the priorities order.

Step 4.2 : the broker agent searches the seller agents that can meet all the constrains starting from the higher priority buyer agent.

Step 4.3 : the broker agent sends OK? message to seller agents with priority from high to low, and each seller agent checks its agent_view to find whether its variable values can meet all the constrains. If all the conditions are met, the seller agent sends the message of matching success to the broker agent. Otherwise, the Nogood message needs to be sent to the broker.

Step 4.4 : repeat Step 4.1-4.4 until all the buyer agents have performed the asynchronous backtracking operation. 
Step 5 : when there are multiple seller agents or no seller agent in the searching resulting, the broker agent negotiates dynamically with buyer agents using multiagent interaction language and method.

(1) For the no seller agent result, the broker agent negotiates with the buyer agent by sending the relaxing constraints' message. Returning to Step 4 if the buyer agent replies and relaxes its constraints. Otherwise, the matching processes finish and the buyer backs to the matching buyers queue waiting for the inclined sellers to match, i.e., Step 1.

(2) For the result of multiple seller agents, the broker agent negotiates with the buyer agent in line with their negotiation behavior, i.e., maximizing the evaluation function $E\left(A_{i}\right)$. The matching results are sent to the seller and buyer agent, respectively, and the matching can be achieved if both sides reply. Otherwise, the buyer returns to the matching buyers queue waiting for the inclined sellers to match, i.e., Step 1, and the seller seeks for the inclined buyers in the matching buyers queue to match, i.e., Step 3.

The interaction and communication in the above noted algorithm can be designed and achieved using KQML (Knowledge Query And Manipulation Language). The critical KQML primitives in this algorithm are given in Algorithm 1.

\section{Multiagent System of Dynamic Supply- Demand Matching Approach}

In dynamic supply-demand matching, the objects are autonomous or semiautonomous, and the processes are changing dynamically. Each object has its multiattribute values or requirements and can be assigned to match with certain cooperators in line with its organizational roles. This complies with the characteristics of multiagent system including autonomy, interactivity, reactivity, cooperation, and adaptability. Thus, the objects and organizational roles can be abstracted as agents.

Multiagent system fixates on the coordination and communication among agents to collaboratively accomplish the supply-demand matching. Each agent is responsible for one or more activities in the dynamic supply-demand matching multiagent system, and each agent interacts with other agents when planning and performing their responsibilities.

5.1. Multiagent System Architecture. This paper designs the architecture of dynamic supply-demand matching multiagent system as two types of agents, i.e., physical agents and logical agents. A physical agent represents tangible existing objects, inclusive of the buyer, seller, and broker. A logical agent represents a logical object with the core function of the system, inclusive of information agent (IA), communicating agent (CA), filtrating agent (FA), negotiation agent (NA), and evaluating agent (EA). The agents and architecture in the dynamic supply-demand matching multiagent system are illustrated in Figure 2, in which the agents in the box are logical agents, the solid lines indicate that each agent can directly contact (such as communication agent and broker agent), and the dotted lines indicate that the contact between agents with the help of a third-party agent, i.e., the communication between the broker agent and seller agent using CA:

(1) The broker agent: this agent represents the broker enterprise, who develops the dynamic supply-demand matching system, and has the authority to manage the system and database. It is the dominant power of supply-demand transaction and can trigger the supply-demand matching tasks by communicating, negotiating, and interacting with other agents.

(2) The buyer agent: this agent represents the buyer to negotiate with other agents and can drive the supplydemand matching. It is capable of releasing demand information, modifying or providing matching parameters, and accepting its own matching results.

(3) The seller agent: this agent represents the seller and guarantees the supply-demand matching. It can supply commodities/services and accept its own matching results.

(4) The information agent (IA): this agent is charged with tasks analyzing and processing in dynamic supply-demand matching. It can store and provide information of other agents and trigger or terminate the corresponding operations.

(5) The communicating agent (CA): this agent is charged with registering and deregistering of all agents that participate in dynamic supply-demand matching. It can form the interaction among the agents by transmitting information.

(6) The filtrating agent (FA): this agent is responsible for asynchronous backtracking of sell agents based on constraint satisfaction problem in accordance with the information of the buyer agent in IA.

(7) The negotiating agent (NA): this agent is capable of negotiating with the buyer agent on behalf of the broker agent when conflicts raise in FA.

(8) The evaluating agent (EA): this agent is the negotiation rules and basis. It can evaluate sell agents in IA filtrating by FA on the basis of the predetermined evaluation function.

5.2. System Function Design and Implementation. Based on the above analysis, the function modules of the dynamic broker dominant supply-demand matching system is shown in Figure 3.

5.2.1. Connecting Module. The connecting module consists of five parts, i.e., user login, user registration, user name, keyword, and verification code. The unregistered users 
(1) tell//Transmitting knowledge or data from sender to receiver.

(2) achieve//The relevant operation of the receiver requiring by the sender.

(3) broadcast//Broadcasting to all the agents in the system.

(4) search $(A, B, D) / /$ Searching receiver B whose variable values can meet the requirements of constrain $D$ that pertains to sender $A$.

(5) result $(A, B, D) / / T h e$ result whether the variable values of receiver $B$ can meet the requirements of constrain $D$ that pertains to sender $A$.

Algorithm 1: The critical KQML primitives.

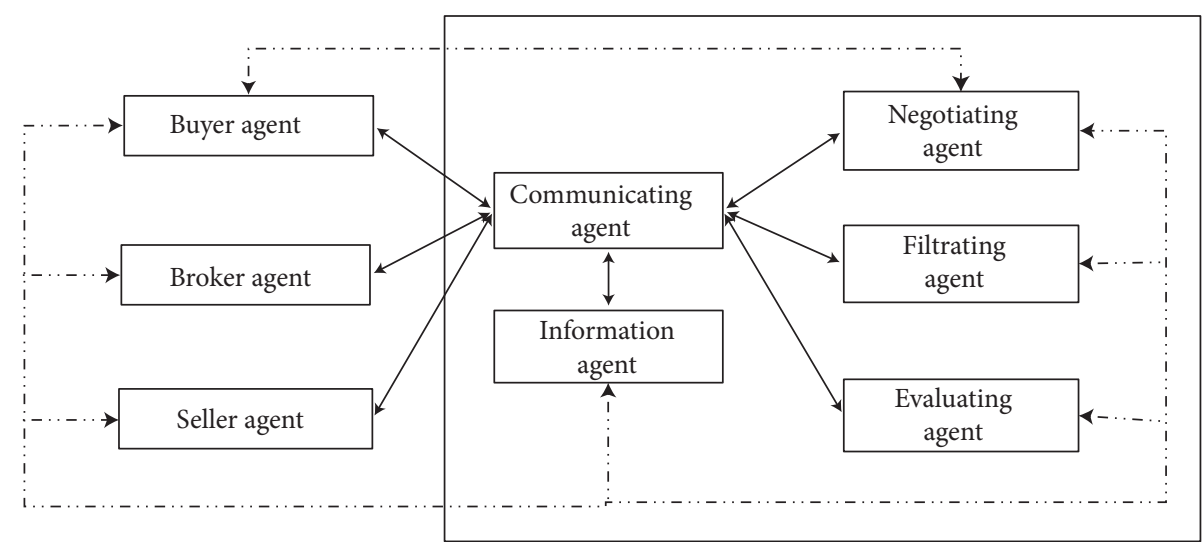

FiguRE 2: Overall framework of the dynamic broker dominant supply-demand matching system.

access system permission by registering, and the registered users login the system by inputting the correct user name, keyword, and verification code. Besides, given that permissions vary with users, their system are also varying with interfaces and functions.

5.2.2. Broker Agent Module. The broker agent, the dominant player of dynamic B-SDMP, serves as the administrator. Its functions and permissions include publishing or viewing information in real time, adding or viewing other agents' basic information, relevant parameters, and supply-demand conditions, and conducting supply-demand matching operations.

5.2.3. Buyer Agent Module. Buyer agent is the driving force for dynamic supply-demand matching, and the major aim of its participation in matching is to seek out the appropriate seller meeting their requirements. Its functions and permissions are to check or modify basic information, add requirements, and check purchase information and match status.

5.2.4. Seller Agent Module. Seller agent can ensure dynamic supply-demand matching, and its participation in matching business is to sell products/services for own profit. Seller agent's functions and permissions comprise viewing or modifying its basic information, viewing demand information and status, submitting or viewing cooperative applications, and matching results.
5.2.5. IA and CA Module. The information agent (IA) is charged with storing and extracting various tasks and data, and the communication agent (CA) is in charge of receiving or sending messages between agents. Both of them should run the processes and operations of dynamic supply-demand matching, which is the vital support for the matching system to operate.

The IA receives the demand information of the buyer released by the CA. Then, the information is passed to the broker agent by the CA. The broker receives demand information from IA and distributes it to the sellers through CA. After the sellers submit the cooperation application, the CA transfers the relevant information to IA for storage. According to the CA's information about sellers and buyers received from the IA, the broker is capable of operating the supply-demand matching including the screening, negotiating, and evaluating of the agents. The cooperation of IA and CA is needed in the information storage and transfer processes.

5.2.6. FA Module. In the dynamic B-SDMP, the broker triggers FA by IA and CA. In this process, taking the buyers' requirements as the domains and sellers' relevant data as the variables, the dynamic B-SDMP multiagent model and its algorithm is employed to find the sellers who meet the requirements of buyers.

5.2.7. NA and EA Module. The negotiating agent (NA) will be invoked in two cases. When there are multiple sellers meeting the requirements, it is necessary to evaluate them by calling for EA, and the seller who is at the top places is 


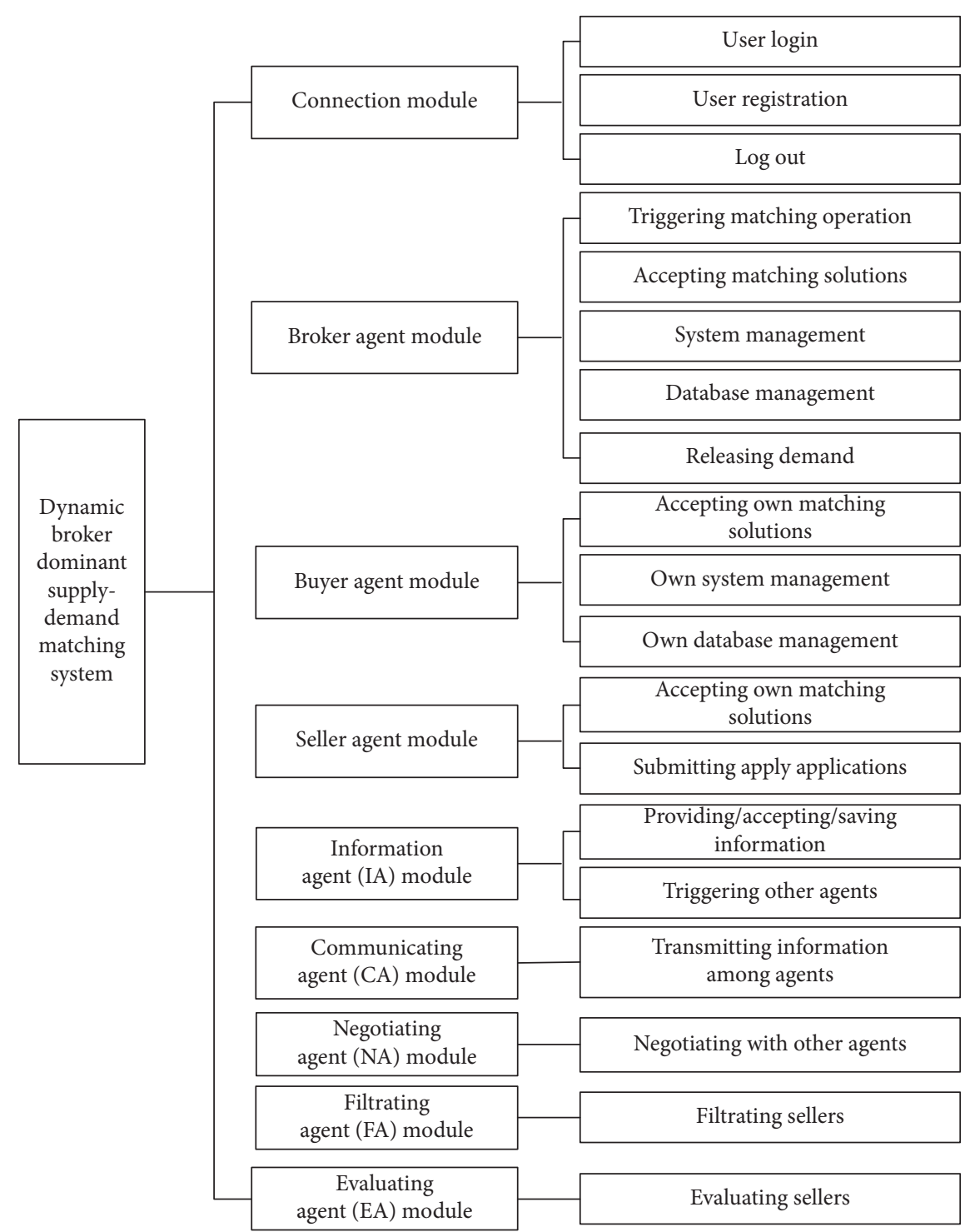

FIGURE 3: Function modules of the dynamic broker dominant supply-demand matching system.

selected as the matching cooperator. In this case, the relevant data for evaluating are obtained through the negotiation between the buyer and the broker using NA. Besides, when there is no seller satisfying all the requirements, the broker should call for NA to negotiate with the buyer agent to amend the parameters and relax the constraint conditions. The data obtained from the negotiation agent are stored and transmitted by IA and CA for relevant calculation.

\section{System Test and Example Simulation}

6.1. System Test Environment. The dynamic broker dominant supply-demand matching system is developed by MyEclipse 10.0 using the browser/server $(\mathrm{B} / \mathrm{S})$ under windows operation system. The data are stored in the database and operated under MySQL coding. In this system, the server side employs JDK and Tomcat 9.0 to connect with the database, and the browser-side performs the relevant operations by inputting the server address.
6.2. Example Design. Considering the influencing factors of the broker in cooperation with sellers, such as quality and price, production capacity, service accuracy, customer concern, and the factors of cooperation relationship, demand level when cooperating with buyers, and other factors, such as scale and investment, historical cooperation, matching degree, technical ability and management level, and market reputation, this paper chooses fourteen criteria as a basis for matching. Assume that buyer $G_{1}$ releases demand information including demand quantity, ideal price, reservation price, and requirements according to fourteen criteria (see Table 1) by the dynamic supply-demand matching system. In Table 1, $C_{1}$ to $C_{14}$, respectively, represent sales profit rate, production and operation capacity, enterprise scale, input and output situation, accuracy rate, reliability, cooperative trading volume, cooperative growth rate, enterprise informatization level, enterprise management level, market reputation, cultural compatibility, target consistency, and information platform matching degree. 
TABLe 1: Demand information of buyer $G_{1}$.

\begin{tabular}{|c|c|c|c|c|c|c|c|c|c|}
\hline Demand information & Value & Index & Value & Index & Value & Index & Value & Index & Value \\
\hline Demand quantity & $1658000 t$ & $C_{1}$ & 20 & $\mathrm{C}_{5}$ & 800000 & $\mathrm{C}_{9}$ & 80 & $C_{13}$ & 70 \\
\hline Ideal price & $¥ 500$ & $\mathrm{C}_{2}$ & 80 & $C_{6}$ & 75 & $C_{10}$ & 83 & $C_{14}$ & 0.00 \\
\hline Reservation price & $¥ 530$ & $\begin{array}{l}C_{3} \\
C_{4}\end{array}$ & $\begin{array}{c}180 \\
60\end{array}$ & $\begin{array}{l}C_{7} \\
C_{8}\end{array}$ & $\begin{array}{l}60 \\
85\end{array}$ & $\begin{array}{l}C_{11} \\
C_{12}\end{array}$ & $\begin{array}{l}85 \\
6.5\end{array}$ & & \\
\hline
\end{tabular}

The broker accepts and reviews the information of buyer $G_{1}$ and releases the relevant data to the sellers. The sellers view these demand information, submit cooperation application, and provide their own supply information, which includes supply quantity, ideal price, reservation price (Table 2), and variable values of fourteen criteria (Table 3).

The broker accepts and reviews the information of the sellers and performs supply-demand matching operation for the approved applications.

6.3. System Running Results. For the dynamic B-SDMP of buyer $G_{1}$ and sellers $A_{1}, A_{2}$, and $A_{3}$, the system running processes and results are as follows.

By taking the data in Table 1 as constraints and data in Table 3 as variable values, the filtrating agent (FA) performs the distributed constraint satisfaction operation for each index. The results suggest that no seller meets the requirements. The broker should negotiate with buyer agent $G_{1}$ by NA, asking for relaxing the requirements.

The buyer agent $G_{1}$ registers the system, views this information in its backlog, and submits the modified requirements.

The broker continues to perform supply-demand matching operation for buyer $G_{1}$, calls $\mathrm{FA}$ again, and conducts asynchronous backtracking based on new constrains. There are three sellers meeting the requirements, and the broker should negotiate with buyer agent $G_{1}$ by NA based on the determined buyer agents negotiating behavior using EA:

(1) Assume that the importance of sellers $A_{1}, A_{2}$, and $A_{3}$ are $0.683,0.383$, and 0.437 , respectively.

(2) Calculate the transaction price and its satisfaction of buyer $G_{1}$ on sellers $A_{1}, A_{2}$, and $A_{3}$, as listed in Table 4.

(3) Calculate comprehensive satisfaction of buyer $G_{1}$ according to its satisfaction about transaction price and preference ordinal, as listed in Table 5.

(4) Choose seller $A_{3}$ whose evaluation function $E\left(A_{3}\right)$ is the greatest as the matched partner of buyer $G_{1}$. The matching results are shown in the system of buyer $G_{1}$ and seller $A_{3}$, respectively.

Base on the noted processes, the dynamic broker dominant supply-demand matching can be achieved by the filtration, evaluation, and negotiation of sellers and buyers.

The above computational results suggest that the dynamic broker dominant supply-demand matching multiagent system can achieve data searching and negotiating among agents based on specific requirements and yield
TABle 2: Basic supply information of sellers $A_{1}, A_{2}$, and $A_{3}$.

\begin{tabular}{lccc}
\hline & $A_{1}$ & $A_{2}$ & $A_{3}$ \\
\hline Supply quantity & $5000000 \mathrm{t}$ & $8000000 \mathrm{t}$ & $10000000 \mathrm{t}$ \\
Ideal price & $¥ 540$ & $¥ 542$ & $¥ 525$ \\
Reservation price & $¥ 520$ & $¥ 525$ & $¥ 510$ \\
\hline
\end{tabular}

TAвLe 3: Variable value of sellers $A_{1}, A_{2}$, and $A_{3}$.

\begin{tabular}{lccc}
\hline & $A_{1}$ & $A_{2}$ & $A_{3}$ \\
\hline$C_{1}$ & 36.16 & 33.34 & 22.84 \\
$C_{2}$ & 92.2 & 96.4 & 81.2 \\
$C_{3}$ & 509 & 510 & 301.6 \\
$C_{4}$ & 64.8 & 70.6 & 62 \\
$C_{5}$ & 1593462 & 2672142.8 & 162865.8 \\
$C_{6}$ & 83.6 & 89.8 & 82.6 \\
$C_{7}$ & 224 & 364.8 & 64.8 \\
$C_{8}$ & 84.32 & 83.22 & 80.05 \\
$C_{9}$ & 90.2 & 78 & 84.4 \\
$C_{10}$ & 83.8 & 97.6 & 89.2 \\
$C_{11}$ & 92.8 & 95.8 & 87 \\
$C_{12}$ & 15.78 & 16.22 & 8.7 \\
$C_{13}$ & 88.6 & 90.6 & 81 \\
$C_{14}$ & 0.13 & 0.02 & 0.12 \\
\hline
\end{tabular}

TABle 4: Transaction price and its satisfaction of buyer $G_{1}$ on sellers.

\begin{tabular}{cccc}
\hline & $A_{1}$ & $A_{2}$ & $A_{3}$ \\
\hline$G_{1}$ & {$[521.38,0.286]$} & {$[526.51,0.251]$} & {$[514.3,0.360]$} \\
\hline
\end{tabular}

TABLe 5: Comprehensive satisfaction of buyer $G_{1}$.

\begin{tabular}{cccc}
\hline & $A_{1}$ & $A_{2}$ & $A_{3}$ \\
\hline$G_{1}$ & 0.2902 & 0.2357 & 0.4020 \\
\hline
\end{tabular}

dynamic matching solutions that can verify the validity and usability of the system functions.

\section{Conclusion}

The aim of dynamic B-SDMP is to seek for proper supplydemand cooperation solutions under uncertainty to improve the formulation of long-term partnership among the sellers, buyers, and broker.

Multiagent systems are initiatives of modern artificial intelligent advances which are developed to meet the requirements of the new world. In this paper, one of the applications of these systems in dynamic B-SDMP modeling was researched, and an information system based on multiagent and Internet technology which is capable of 
achieving the negotiation and interaction among agents was established.

The primary contribution of this paper is as follows:

(1) The process of defined dynamic supply-demand matching, which stresses the environmental dynamics and the leading role of the broker in contrast with the existing SDMP in the literature.

(2) The formalization of the proposed dynamic B-SDMP decision model and its algorithm along with

The proposed dynamic supply-demand matching multiagent model, i.e., the distributed constraint satisfaction model based on dynamic negotiation. The proposed asynchronous backtracking algorithm based on the multiagent interaction method using knowledge query and manipulation language.

(3) The design and development of the dynamic matching system in accordance with multiagent using Java Web and KQML.

However, it still needs much more further works to improve and enrich the models, and it also brings potential directions on broker dominant supply-demand matching. Some outlooks on further works are pointed out as follows:

(1) The influence of broker's interest and preference on supply-demand matching

(2) What are the new characteristics of supply-demand matching problem and how to model it if there are multiple brokers and competitions among them.

\section{Data Availability}

The generated and analyzed data used to support the findings of this study are included within the article.

\section{Conflicts of Interest}

The authors declare that they have no conflicts of interest.

\section{Acknowledgments}

This work was partly supported by the Doctor Foundation Project of Yanshan University (Project no.8190088) the Scientific, Technological Research Projects of Colleges and Universities in Hebei Province (Project no. QN2019150) and Youth program of Natural Science Foundation of Hebei Province (Project no.G2020030006).

\section{References}

[1] A. Bhattacherjee, "Acceptance of e-commerce services: the case of electronic brokerages," IEEE Transactions on Systems, Man, and Cybernetics-Part A: Systems and Humans, vol. 30, no. 4, pp. 411-420, 2000.

[2] T.-P. Liang and J.-S. Huang, "A framework for applying intelligent agents to support electronic trading," Decision Support Systems, vol. 28, no. 4, pp. 305-317, 2000.
[3] T. L. Tyrone and I. L. Hsuan, "Analysis of required and matching loan qualities in financial institutions," Journal of Statistics and Management Systems, vol. 9, no. 1, pp. 105-22, 2006.

[4] T.-C. Chiang and F.-J. Yu, "Improving real estate broker service using TOPSIS method," Journal of Information and Optimization Sciences, vol. 30, no. 2, pp. 231-243, 2009.

[5] F. F. S. Ming, L. Ming, and M. A. Simaan, "Resource supplydemand matching scheduling approach for construction workface planning," Journal of Construction Engineering and Management, vol. 142, no. 1, 2014.

[6] J. J. Jung and G. S. Jo, "Brokerage between buyer and seller agents using constraint satisfaction problem models," Decision Sup-Port Systems, vol. 28, no. 4, pp. 293-304, 2001.

[7] L. Klerkx and C. Leeuwis, "Matching demand and supply in the agricultural knowledge infrastructure: experiences with innovation intermediaries," Food Policy, vol. 33, no. 3, pp. 260-276, 2008.

[8] Z.-Z. Jiang, W. H. Ip, H. C. W. Lau, and Z.-P. Fan, "Multiobjective optimization matching for one-shot multi-attribute exchanges with quantity discounts in e-brokerage," Expert Systems with Applications, vol. 38, no. 4, pp. 4169-180, 2011.

[9] H. M. Liang and Y. P. Jiang, "A method for buy-sell two-sided matching decision-making method considering the trade attitude of the broker," Operations Research and Management Science, vol. 22, no. 5, pp. 128-133, 2013.

[10] A. A. Amrutha, M. Mathirajan, and P. Balachandra, "A mathematical model for supply demand matching in a low carbon electricity system," Journal of Information and Optimization Sciences, vol. 37, no. 5, pp. 763-790, 2016.

[11] X. Zhang, X.-M. Yuan, J.-G. Yuan, and H.-J. Hu, "Multiobjective approach for broker dominant supply-demand matching decision," Journal of Interdisciplinary Mathematics, vol. 20, no. 4, pp. 1139-1152, 2017.

[12] X. Wang, Y. Liu, and Y. Nan, "A stable-matching-based user linking method with user preference order," Mathematical Problems in Engineering, vol. 2017, Article ID 3247627, 8 pages, 2017.

[13] H. Zhou, J. C. Dong, and Y. Lean, "An agent-based model for investigating the impact of distorted supply-demand information on China's resale housing market," Journal of Computational Science, vol. 25, no. 3, pp. 1-15, 2018.

[14] G. Zaefarian, Understanding the Interplay between Business Relationships and Business Strategy Using Configuration Theory, University of Manchester for The Degree of Doctor, Manchester, UK, 2011.

[15] S. T. Wang, "An analysis of manufacturers' supply and demand uncertainty based on the dynamic customisation degree," International Journal of Production Research, vol. 6729, no. 49, pp. 3023-3043, 2011.

[16] H. Xu, Y. C. Feng, and S. R. Xu, "A Study, based on the perspective of the dynamic fit, on the construction and the evolution, on the relationship between the supplier and the key customer: a case study on Lishen group developing 12 key customers," Management World, vol. 30, no. 4, pp. 107-123, 2014.

[17] W. X. Jiang, X. Z. Hu, M. X. Liu et al., "Information collaboration model of cloud computing supply chain based on multi-agent," Journal of System Simulation, vol. 28, no. 1, pp. 51-56, 2016.

[18] W. Xu, S. Guo, X. Li, C. Guo, R. Wu, and Z. Peng, “A dynamic scheduling method for logistics tasks oriented to intelligent manufacturing workshop," Mathematical Problems in Engineering, vol. 2019, Article ID 7237459, 18 pages, 2019. 
[19] H. Dastyar, D. Rippel, and M. Freitag, "Optimization of supplier development under market dynamics," Mathematical Problems in Engineering, vol. 2020, Article ID 2912380, 18 pages, 2020.

[20] Z. P. Fan and Q. Yue, "Strict two-sided matching method based on complete preference ordinal information," Journal of Management Sciences in China, vol. 17, no. 1, pp. 21-34, 2014. 